\title{
УДК (UDC) 621.432.3
}

\author{
ВЛИЯНИЕ РЕЖИМА РАБОТЫ ДВИГАТЕЛЯ \\ ТРАНСПОРТНО-ТЕХНОЛОГИЧЕСКОЙ МАШИНЫ НА БИЕНИЕ В \\ СОПРЯЖЕНИИ ПОРШЕНЬ-ЦИЛИНДР
}

\section{THE INFLUENCE OF THE MODE OF ENGINE OPERATION OF TRANSPORT AND TECHNOLOGICAL CARS ON THE BEATING AT THE COUPLING PISTON-CYLINDER}

\author{
Штайн Г.В., Панфилов А.А. \\ Shtain G.V., Panfilov A.A. \\ Тюменский индустриальный университет (Тюмень, Россия) \\ Tyumen Industrial University (Tyumen, Russian Federation)
}

\begin{abstract}
Аннотация. Перекладка или биение поршня возникает вследствие действия боковой силь в кривошипно-цатунном механизме и приводит к увеличению сил трения и ударных нагрузок в сопряжении с гильзой. В конечном итоге это влияет на работу двигателя, его иумность, вибрацию и вызывает интенсивное изнашивание юбки пориня. Поэтому исследование вопросов, связанных с повышением надежности двигателя транспортно-технологических машин, является актуальной задачей технической эксплуатации.Целью работы является повышение надежности двигателя транспортнотехнологических машин путем снижения вибрации при его работе. В статье рассмотрен способ, позволяюший уменьшить ударные нагрузки в сопряжении поршень-цилиндр на малой частоте вращения коленчатого вала при максимальном вращающеем моменте, что характерно для основных режимов двигателей транспортно-технологических $u$ дорожно-строительных машина. Предлагается на режимах максимальных нагрузок при минимальной частоте вращения обеспечить величину суммарной сильг при прохождении через верхнюю мертвую точку в конце такта сжатия равной нулю. Для того, чтобы сила давления газов в верхней мертвой точке была равной силе инерции поступательно движушихся масс, рекомендуется выполнить тюнинг двигателя по изменению фаз газораспределения на такте сжатия, т.е. увеличить угол запаздывания закрытия впускного клапана. Проведен расчет по определению угла запаздывания закрытия впускного клапана на примере двигателя ЯМЗ-65202.10. Для выполнения равенства силь давления газов и силь инерции в верхней мертвой точке найдена величина реальной степени сжатия двигателя, которая составила 12,5. Далее определён угол запаздывания закрытия впускного клапана для данного режима, который по расчету равен $54^{0}$ поворота коленчатого вала после нижней мертвой точки. Расчетные данные могут являться исходными параметрами для калибровки программы, заложенной в электронный блок управления транспортнотехнологической машины.
\end{abstract}

Abstract. Relocation or beating of the piston occurs due to the action of lateral force in the crank mechanism, which leads to an increase in friction forces and shock loads in conjunction with the sleeve. Ultimately, this affects the operation of the engine, its noise, vibration and leads to intensive wear of the piston skirt. Therefore, the study of issues related to improving the reliability of the engine of transport and technological vehicles is an urgent task of technical operation. The aim of the work is to increase the reliability of the engine of transport-technological machines by reducing vibration during its operation. The article discusses a method that allows to reduce shock loads in the piston-cylinder coupling at a low crankshaft rotational speed at maximum torque, which is typical for the main engine modes of a transport-technological and road-building machine. It is proposed that under maximum load conditions with a minimum speed of rotation, the value of the total force when passing through the top dead center at the end of the compression stroke be equal to zero. In order for the gas pressure force at the top dead center to be equal to the inertia force of the translationally moving masses, it is recommended that the engine be tuned to change the valve timing during a compression stroke, i.e. increase the angle of delay in closing the intake valve. In confirmation of the above, a calculation was made to determine the angle of delay in closing the intake valve using the example of the YaMZ-65202.10 engine. To fulfill the equality of the gas pressure force and the inertia force at the top dead center, the value of the real engine compression ratio was found, which was 12.5 . Next, the angle of the inlet valve closing lag is determined for this mode, which is calculated to be $54^{0}$ crankshaft rotation after bottom dead center (according to the technical characteristics of this engine, this value is $48^{\circ}$ ). The calculated data may be the initial parameters for the calibration of the program embedded in the electronic control unit of the transport and technological machine. 
Ключевые слова: дорожно-строительная машина, биение поршня, боковая сила, фазы газораспределения, реальная степень сжатия, чип-тюнинг.

Дата принятия к публикации: Дата публикации:

Сведения об авторах:

Штайн Геннадий Вольфович - кандидат технических наук, доцент кафедры сервиса автомобилей и технологических машин, Тюменский индустриальный университет, e-mail: gena.shtain@yandex.ru

Панфилов Александр Анатольевич кандидат технических наук, доцент кафедры сервиса автомобилей и технологических машин, Тюменский индустриальный университет,

e-mail: panfilovaa72@yandex.ru

\section{1. Введение}

Для современных двигателей транспортно-технологических машин, оснащенных средствами глушения шума систем впуска и выпуска, преобладающее значение на шумовые характеристики влияют вибрации сопряженных деталей и узлов, таких как блоккартер на опорах, приводы навесного оборудования, механизма газораспределения $[3,9]$.

Существенное значение в шумовые характеристики двигателя оказывает биение поршня в сопряжении с цилиндром [8]. Перекладка поршня и соответствующее биение, сопровождающее шумом [2], возникает в момент изменения направления действия боковой силы $F_{N}$ (рис.1).

На интенсивность удара поршня по цилиндру оказывает влияние частота изменения величины силы $F_{N}$ в момент соприкосновения, величина зазора в сопряжении, масса поршневой группы, длина юбки поршня [1]. В четырехтактном двигателе момент изменения знака боковой силы и количество перекладок определяются закономерностью изменения сил инерции $F_{a}$ и давления газов в цилиндре $F_{2}$.

Сила инерции определяется по формуле

$$
F_{a}=-m_{n} a_{n},
$$

где $m_{n}$ - масса деталей кривошипно-шатунного механизма, движущихся поступательно (в нее включают поршневую группу цилиндра двигателя и часть массы шатунной груп-
Keywords: road-construction machines, piston beating, lateral force, valve timing, real compression ratio, chip tuning.

Date of acceptance for publication:

10.10.2019

Date of publication:

25.12.2019

\section{Authors' information:}

Gennady V. Shtain - Candidate of Technical Sciences, Associate Professor at the Department of Automobile Service and Technological Machines, Tyumen Industrial University, e-mail: gena.shtain@yandex.ru

Alexander A. Panfilov - Candidate of Technical Sciences, Associate Professor at the Department of Automobile Service and Technological Machines, Tyumen Industrial University,

e-mail:panfilovaa72@yandex.ru.

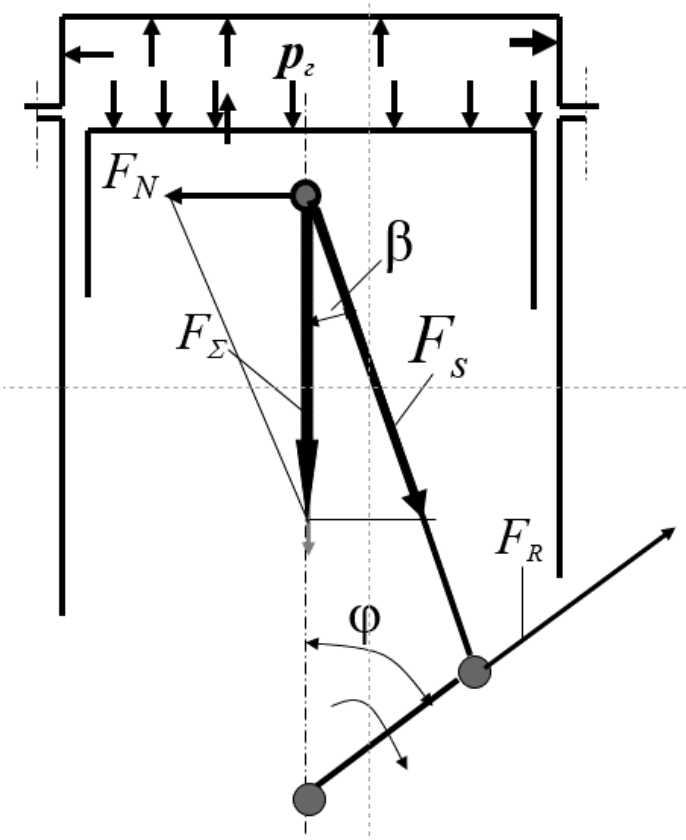

Рис. 1. Силы, действующие на детали кривошипно-шатунного механизма двигателя

пы, движущейся поступательно, при динамическом расчете эту массу принимают равной $\left.(0,2 \ldots 0,3) m_{u}\right) ; a_{n}$ - ускорение поршня.

Ускорение поршня определяется из уравнения

$$
a_{n}=r \omega^{2}(\cos \varphi+\lambda \cos 2 \varphi),
$$

где $r$ - радиус кривошипа коленчатого вала; $\lambda$ - параметр критерия кинематического подобия кривошипно-шатунного механизма (для большинства автотракторных двигателей эта величина составляет $\lambda=0,24 \ldots 0,31)$.

Параметр $\lambda$ можно определить как 


$$
\lambda=\frac{r}{l_{ш}}
$$

где $l_{w}$ - длина шатуна.

Силы, действующие на детали кривошипно-шатунного механизма двигателя, представлены на рис. 1. Суммарная сила, действующая на поршень по оси цилиндра, определяется как сумма силы давления газов и силы инерции:

$$
F_{\Sigma}=F_{2} \pm F_{a} .
$$

Боковая сила $F_{N}$, действующая в сопряжении поршень-цилиндр, является составляющей от суммарной силы:

$$
F_{N}=F_{\Sigma} \operatorname{tg} \beta .
$$

Сила, действующая вдоль шатуна, также является составляющей суммарной силы:

$$
F_{S}=F_{\Sigma} / \cos \beta \text {. }
$$

Центробежная сила инерции вращающихся масс кривошипно-шатунного механизма определяется по формуле:

$$
F_{R}=F_{\Sigma} \cos (x+\beta) \frac{1}{\cos \beta} .
$$

Сила давления газов, действующая на поршень, определяется зависимостью:

$$
F_{\Gamma}=p_{\Gamma} \frac{\pi \cdot D^{2}}{4} .
$$

Центробежная сила передается на опоры двигателя, вызывая переменные по величине реакции. Численные значения тригонометрических функций, входящих в приведенные выше уравнения для различных углов $\varphi$ и $\beta$, принимаются в соответствии со справочными данными.

Для двигателей транспортно-технологических машин, работающих как строительно-дорожная техника, основным режимом работы является режим максимального вращающего момента, где частота вращения составляет, как правило, величину $1100 \ldots 1400$ мин $^{-1}$ (рис. 2).

При такой относительно малой частоте вращения сила инерции $F_{a}$ составляет незначительную величину и суммарная сила, действующая на поршень $F_{\Sigma}$, усугубляет ударное воздействие поршня на стенку цилиндра.

Как видно из рис. 3, вблизи верхней мертвой точки боковая сила $F_{N}$ является знакопеременной, что приводит к усилению интенсивности ударов поршня по цилиндру [11].

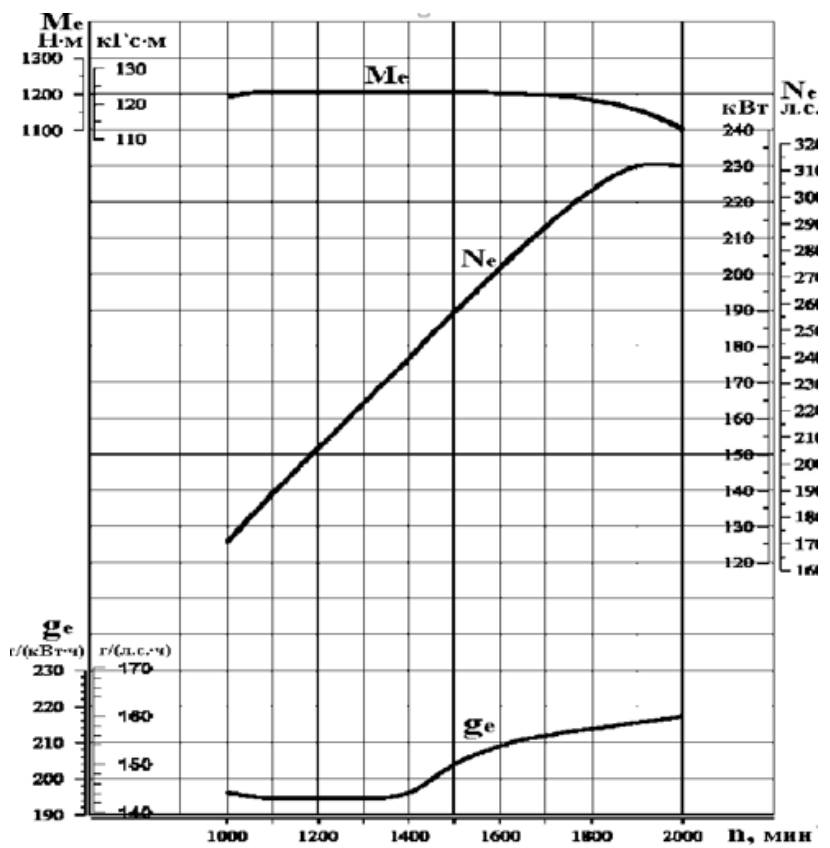

Рис. 2. Эксплуатационная характеристика двигателя ЯМЗ-6502.10

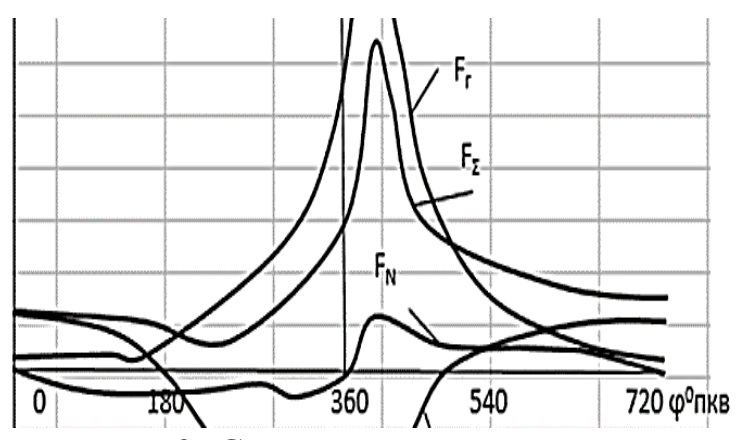

Рис. 3. Силы, действующие в кривошипно-шатунном механизме в режиме максимального вращающего момента

Необходимо учитывать и такой неблагоприятный фактор для сопряжения поршеньцилиндр при действии боковой силы как усиленный износ юбки поршня (рис. 4) с негативными последствиями [4].

Таким образом, конструктивные и технологические особенности поршневых двигателей, применяемых на транспортно-технологических машинах, существенно влияют как на шумовые характеристики транспортного средства, которые являются одним из показателей его экологической безопасности, так и на такой существенный фактор, как надежность энергоустановки. 


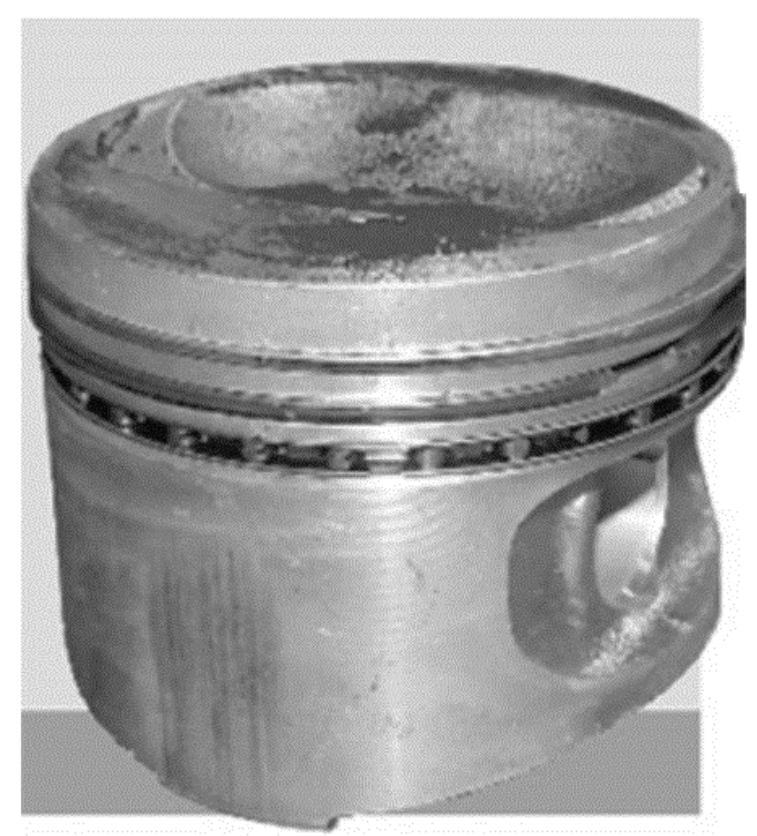

Рис. 4. Характерный износ юбки поршня от воздействия боковой силы

\section{2. Теоретические исследования}

Из практики эксплуатации двигателей транспортно-технологических машин известно, что при повышении частоты вращения, сохранении нагрузки и росте сил инерции суммарная сила на участке перед верхней мертвой точкой становится отрицательной и перекладка поршня исключается. Этот благоприятный фактор учитывают квалифицированные механизаторы и водители строительно-дорожных машин при выполнении соответствующих работ.

В практике проектирования и конструирования поршневых двигателей большой мощности (судовые, стационарные энергоустановки) применяются дезаксиальный (смещенный) кривошипно-шатунный механизм (рис. 5.). В результате смещения оси цилиндра относительно оси коленчатого вала на такте расширения боковая сила $F_{N}$ уменьшается, а на такте сжатия увеличивается, в результате чего обеспечивается равномерный износ цилиндра. Это происходит вследствие того, что суммарная сила $F_{\Sigma}$ не проходит через ось коленчатого вала. Таким образом, создается дополнительный вращающий момент, который уменьшает воздействие боковой силы на стенку цилиндра на такте расширения, а на такте сжатия этот момент ока- зывает обратное действие. Кроме того, необходимо учитывать, что ускорение поршня в дезаксиальном механизме несколько увеличивается по сравнению с ускорением в центральном механизме на величину е $\lambda \sin \varphi$ :

$$
a_{n}=r \omega^{2}(\cos \varphi+\lambda \cos 2 \varphi)+e \lambda \sin \varphi, \quad(6)
$$

где $e$ - смещение оси поршня относительно оси коленчатого вала (рис.5).

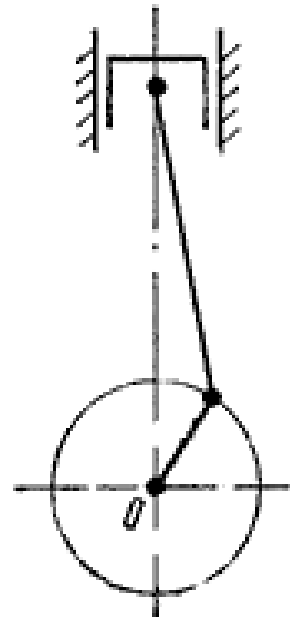

a

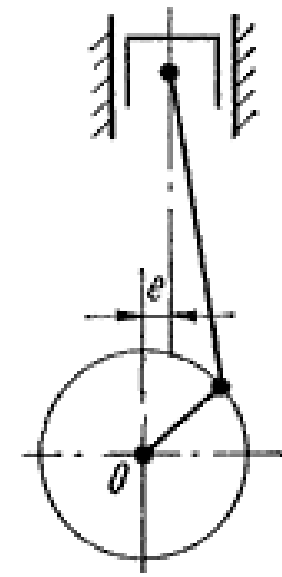

6
Рис.5. Схемы кривошипно-шатунных механизмов поршневого двигателя: а-центральный; б-дезаксиальный

Для двигателей транспортных машин дезаксиальный кривошипно-шатунный механизм не находит применения, так как эта конструкция приводит к увеличению габаритов энергоустановки.

В целях снижения вибрации двигателя транспортно-технологических машин при его работе предлагается на режиме максимальных нагрузок при незначительной частоте вращения коленчатого вала двигателя, когда сила инерции поступательно движущихся масс кривошипно-шатунного механизма является небольшой, обеспечить отрицательной величину суммарной силы $F_{\Sigma}$ на такте сжатия, а при прохождении через верхнюю мертвую точку - равной нулю. Для

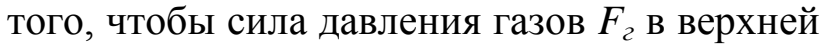
мертвой точке была равна силе инерции поступательно движущихся масс $F_{a}$, необходимо выполнить тюнинг двигателя по изменению фаз газораспределения на такте сжатия, т.е. увеличить угол запаздывания закрытия 
впускного клапана. Указанное инженерное решение по изменению фаз газораспределения для конкретного двигателя должно выполняться с учетом специфики выполняемых работ конкретной транспортно-технологической машины и особенностей конструкции соответствующего двигателя. При этом сочетание изменяющего угла запаздывания закрытия впускного клапана и изменения давления наддува воздуха с учетом калибровки электронного блока управления энергоустановки должно обеспечивать сохранение требуемого коэффициента наполнения и, соответственно, вращающего момента двигателя. Таким образом, для этих двигателей требуется соответствующее программное обеспечение микропроцессоров мехатронной системы управления

У современных двигателей в программном обеспечении электронного блока управления предусмотрена система изменения фаз газораспределения (VVT-Variable Valve Timing) $[5,7,10]$. Данная система предназначена для регулирования параметров работы газораспределительного механизма (изменяет моменты открытия и закрытия впускных и выпускных клапанов). Ее применение позволяет повысить мощность, вращающий момент, топливную экономичность, улучшить пусковые свойства двигателя в зимний период, уменьшить выброс токсичных газов. Для двигателей устаревшей конструкции, где нет системы «VVT-Variable Valve Timing», можно воспользоваться установкой соответствующего угла запаздывания закрытия впускного клапана поворотом газораспределительного вала на расчетный угол против исходного. Необходимо иметь ввиду, что в поршневых двигателях грузовых автомобилей, особенно бензиновых, для снижения шума от ударов поршня по цилиндру в момент его перекладывания в верхней мертвой точке. применялась удлиненная юбка поршня и дезаксаж поршневого пальца. В настоящее время такие конструктивные мероприятия не находят применения, прежде всего, из-за тго, что это отрицательно сказывается на уравновешивании двигателя в целом. Таким образом, прежде, чем выполнять соответствующие настройки газораспредели- тельного механизма конкретного двигателя, необходимо оценить его конструктивные особенности и эксплуатационные характеристики, выполнить в полном объеме его динамический расчет с учетом оптимального для данного режима работы транспортнотехнологичес-кой машины угла запаздывания закрытия впускного клапана.

\section{3. Результаты исследования}

В качестве примера проведем расчет требуемого угла запаздывания закрытия впускного клапана для двигателя ЯМЗ-6502.10, наиболее типовой энергоустановки для транспортно-технологических машин. Его эксплуатационная характеристика представлена на рис. 2. Сила инерции поступательно движущихся масс кривошипно-шатунного механизма данного двигателя на режиме максимального вращающего момента при частоте вращения коленчатого вала $n=1100$ мин $^{-1}$ определится по формуле (1).

При проведении расчетов суммарная масса поршневой группы и части массы шатунной группы, движущихся поступательно по оси цилиндра, принята равной 3,7 кг, а ускорение поступательно движущихся масс кривошипно-шатунного механизма $-13170 \mathrm{~m} / \mathrm{c}^{2}$. Сила $F_{a}=48731 \mathrm{H}$.

Как отмечалось раньше, чтобы суммарная сила $F_{\Sigma}$ в верхней мертвой точке на такте сжатия была равна нулю, необходимо обеспечить равенство сил $F_{a}$ и $F_{2}$. В этом случае величина давления газов в конце такта сжатия определится из следующего выражения

$$
p_{\Gamma}=\frac{4 \cdot F_{\Gamma}}{\pi \cdot D^{2}}
$$

где $D$ - диаметр поршня.

Давление газов составит $p_{2}=4,1 \mathrm{MПа.}$

В общем случае давление газов в конце процесса сжатия рабочего цикла определяется по формуле

$$
p_{\Gamma}=p_{\kappa} \varepsilon^{n_{1}},
$$

где $p_{\kappa}$ - давление наддува турбокомпрессором на данном режиме (принято $p_{\kappa}=$ $0,15 \mathrm{MПа);} \varepsilon$ - геометрическая степень сжатия (принято $\varepsilon=16,4$ ).

Показатель политропы сжатия $n_{1}$ определяется из соотношения:

$$
n_{1}=1,41-100 / n,
$$


где $n$ - частота вращения коленчатого вала двигателя на данном режиме $\left(n=100\right.$ мин $\left.^{-1}\right)$.

Показатель политропы сжатия составит $n_{1}=1,32$.

По результатам расчета величина давления в конце процесса сжатия на данном режиме: $p_{2}=5,85$ МПа. Для того, чтобы получить величину давления газов в конце процесса сжатия равной 4,1 МПа, необходимо воздействовать на величину реальной степени сжатия $\varepsilon$, которая по результатам расчета по формуле (8) должна быть равной 12,5. Необходимо иметь в виду, что для сохранения требуемой величины вращающего момента на данном режиме, необходимо воздействовать на клапан регулирования давления наддува турбокомпрессора за счет соответствующей «прошивки» электронного блока управления.

Потеря реального рабочего объема цилиндра из-за угла запаздывания закрытия впускного клапана оценивается коэффициентом $\psi[6]$ :

$$
\psi=\frac{\Delta V_{h}}{V_{h}}=\frac{s^{\prime}}{S},
$$

где $\Delta V_{h}$ - объем цилиндра, создаваемый поршнем при ходе от нижней мертвой точки до момента закрытия впускного клапана; $V_{h}$ - рабочий объем цилиндра; $S$ - ход поршня (согласно характеристики двигателя ЯМЗ$6502.10 S=0,156$ м).

Ход поршня $S$, при котором на такте сжатия впускной клапан открыт, определяется соотношением:

$$
S^{\prime}=r\left[\left(1-\cos \varphi_{2}\right)+\lambda / 4\left(1-\cos 2 \varphi_{2}\right],\right.
$$

где $\varphi_{2}$ - угол запаздывания закрытия впускного клапана после нижней мертвой точки.

Для двигателя ЯМЗ-6502.10 величина $\lambda=$ 0,27 , ход поршня $S=0,156$ м, радиус кривошипа $r=S / 2=0,078$ м. В этом случае реальная степень сжатия двигателя будет определяться зависимостью

$$
\varepsilon_{\mathrm{p}}=\varepsilon(1-\psi)+\psi,
$$

где $\varepsilon$ - геометрическая степень сжатия (для двигателя ЯМЗ-6502.10 $\varepsilon=16,4)$.
Из этой формулы, принимая $\varepsilon=16,4$, реальная степень сжатия $\varepsilon_{\mathrm{p}}=12,5$, а величина коэффициента $\psi=0,25$.

Из формулы (10) находим величину $S=$ 0,039 м. По формуле (11) определяем величина угла запаздывания закрытия впускного клапана, которая будет равна $54^{0}$ поворота коленчатого вала после нижней мертвой точки (по технической характеристике данного двигателя эта величина составляет $48^{0}$ ). С помощью известных зависимостей, определяющих динамику поршневого двигателя, были определены численные значения (табл. 1) и построены графические зависимости сил $F_{2}, F_{a}, F_{\Sigma}, F_{N}$ (рис. 6).

Сопоставляя графики сил на рис. 3 и рис. 6, действующих на детали кривошипношатунного механизма двигателя ЯМЗ6502.10 , видно, что боковая сила $F_{N}$, действующая со стороны поршня на цилиндр в верхней мертвой точки равна нулю в обоих случаях (в базовом варианте и при тюнинге). В первом случае происходит так называемая перекладка поршня: сила $F_{N}$ меняет знак, что сопровождается биением (рис. 3). Во втором случае сила $F_{N}$ и на такте сжатия, и на такте расширения сохраняет положительное значение своей величины и биение исключается (рис. 6) . Несмотря на рекомендуемые проектные решения по тюнингу данного двигателя, воздействие поршня на цилиндр с левой стороны по ходу вращения коленчатого вала остается и конструктивные меры в сочетании с технологическими мероприятиями по уменьшению износа цилиндропоршневой группы должны быть реализованы. Необходимо особо отметить, что в предлагаемом варианте по изменению угла запаздывании закрытия впускного клапана одновременно требуется корректировать величину давления наддува воздуха во впускном тракте турбокомпрессором, то есть выполнить соответствующую коррекцию программного обеспечения электронного блока управления двигателя. 
Кинематические и динамические параметры двигателя ЯМЗ-6502.10 по результатам расчета

\begin{tabular}{|c|c|c|c|c|c|c|}
\hline$\phi^{0}$, п.к.в. & $a, \mathrm{M} / \mathrm{c}^{2}$ & $F_{a}, \mathrm{H}$ & $F_{2}, \mathrm{H}$ & $F_{\Sigma}, \mathrm{H}$ & $\operatorname{tg} \beta$ & $F_{N}, \mathrm{H}$ \\
\hline 180 & $-754,78$ & 28012,3 & 1781,44 & 29793,8 & 0 & 0 \\
\hline 210 & $-755,84$ & 28051,7 & 11876,3 & 39927,9 & 0,136 & 1615,172 \\
\hline 240 & $-656,55$ & 24366,9 & 23752,5 & 48119,4 & 0,239 & 5676,8547 \\
\hline 270 & $-279,16$ & 10360,7 & 29690,7 & 40051,4 & 0,278 & 8254,0042 \\
\hline 300 & 377,389 & -14006 & 35628,8 & 21622,6 & 0,239 & 8515,282 \\
\hline 330 & 1035 & -38412 & 41566,9 & 3154,53 & 0,136 & 5653,1021 \\
\hline 360 & 1313,11 & -48734 & 48721,8 & $-11,983$ & 0 & 0 \\
\hline 390 & 1035 & -38412 & 142515 & 104103 & 0,136 & 19382,064 \\
\hline 420 & 377,389 & -14006 & 118763 & 104756 & 0,239 & 28384,273 \\
\hline 450 & $-279,16$ & 10360,7 & 95010,1 & 105371 & 0,278 & 26412,813 \\
\hline 480 & $-656,55$ & 24366,9 & 71257,6 & 95624,5 & 0,239 & 17030,564 \\
\hline 510 & $-755,84$ & 28051,7 & 47505,1 & 75556,7 & 0,136 & 6460,6882 \\
\hline 540 & $-754,78$ & 28012,3 & 35628,8 & 63641,1 & 0 & 0 \\
\hline
\end{tabular}

$F, H$

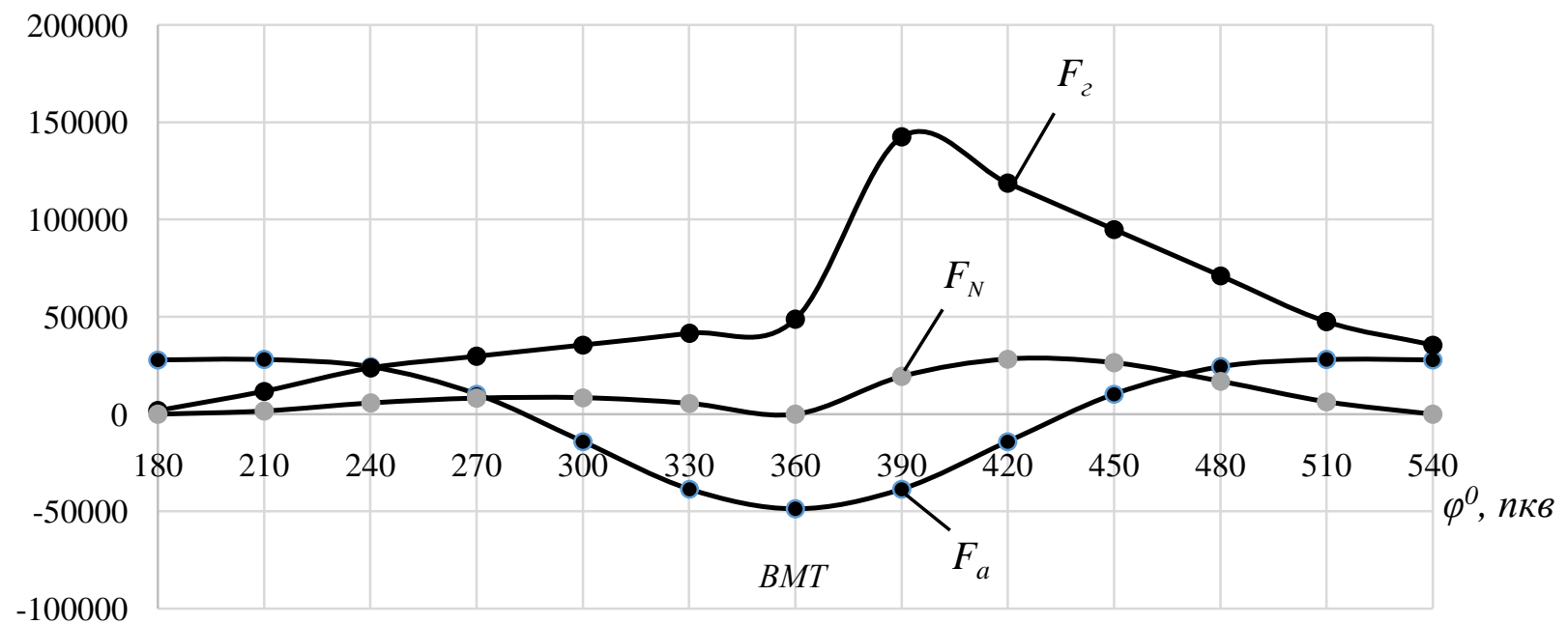

Рис. 6. Силы, действующие в кривошипно-шатунном механизме в режиме максимального вращающего момента с учетом тюнинга двигателя по углу запаздывания закрытия впускного клапана

\section{4. Заключение}

Вибрация (биение) поршня в сопряжении с цилиндром в момент прохождения верхней мертвой точки является одним из основных источников шума, сопровождающего работу двигателя транспортно-технологических машин на рабочем режиме под нагрузкой. Шумовая характеристика двигателя одновременно с выбросом токсичных отработавших газов определяет экологические показатели данного транспортного средства, экс- плуатируемого в населенных пунктах. В существующих и находящихся в эксплуатации поршневых двигателей транспортнотехнологических машин предусматриваются ряд конструктивных и технологических мероприятий, уменьшающих негативное воздействие боковой силы $F_{N}$ в сопряжении поршень-цилиндр. Это прежде всего конструкция и правила установки поршневых колец, износостойкие из специального материала вставки в верхнем поясе цилиндра (гильзы), конструкция верхней головки 
поршня. Причем считается, что перекладывание поршня в верхней мертвой точке и соответствующее биение, сопровождающее износом цилиндра и поршня, является объективным фактором. В инструкциях по эксплуатации транспортно-технологических машин указывается о необходимости кратковременности работы двигателя в режиме максимального вращающего момента.

В данной работе выполнены соответствующие расчеты по тюнингу двигателя ЯМ3-65202.10, имеющего массовое применение в транспортно-технологических машинах, с определением оптимального угла

\section{Список литературы}

1. Балакин П.Д., Бельков В.Н. Динамические реакции в связях механического привода поршневых машин // Материалы II Междунар. научно-техн. конф. Омский государственный технический университет. Омск: ОГТУ, 2018. С. 5-8.

2. Кузнецов В.Н., Беляев В.И., Мельников Ф.П. Влияние фаз газораспределения на изменение давления во впускном коллекторе многоцилиндрового двигателя // Вестник Алтайского государственного аграрного университета. 2014. № 12 (122). С. 137-141.

3. Петров А.П., Казаков Ю.Ф. К вопросу разработки нового типа двигателя внутреннего сгорания // Вестник Чувашской государственной сельскохозяйственной академии. 2018. № 1 (4). С. 93-96.

4. Путинцев С.В., Агеев А.Г. Проверка эффективности применения ребер жесткости юбки поршня малоразмерного дизеля // Тракторы и сельхозмашины. 2016. № 11. С. 35-39.

5. Сазгетдинов К.М., Березовский А.Б., Максимов А.В. Перспективные системы управления газораспределением автомобильных двигателей // В сб.: XXIII Туполевские чтения (школа молодых ученых). 2017. C. 240-246.

6. Штайн Г.В., Панфилов А.А. Эксплуатация транспортно-технологических машин в зимних условиях. Тюмень: ТюмГНГУ, 2016. 68c.

7. Штайн Г.В., Панфилов А.А. Меха- запаздывания закрытия впускного клапана. Это позволяет исключить такой неблагоприятный фактор воздействия боковой силы в режиме максимального вращающего момента, как биение в сопряжении поршеньцилиндр, сопровождающееся существенным шумом и износом цилиндро-поршневой группы.

В дальнейшем возможна разработка программного комплекса по определению углу запаздывания закрытия впускного клапана для современных двигателей транспортнотехнологических машин с учетом снижения вибрации сопряженных деталей.

\section{References}

1. Balakin P.D., Belkov V.N. Dinamicheskie reaktsii v svyazyakh mekhanicheskogo privoda porshnevykh mashin. Materialy II Mezhdunarodnoy nauchno-tekhnicheskoy konferentsii, Omsk State Technical University. Omsk, 2018, pp. 5-8. (In Russian)

2. Kuznetsov V.N., Belyaev V.I., Melnikov F.P. Vliyanie faz gazoraspredeleniya na izmenenie davleniya vo vpusknom kollektore mnogotsilindrovogo dvigatelya. Vestnik Altayskogo gosudarstvennogo agrarnogo universiteta, 2014, No. 12 (122), pp. 137-141. (In Russian)

3. Petrov A.P., Kazakov Yu.F. K voprosu razrabotki novogo tipa dvigatelya vnutrennego sgoraniya. Vestnik Chuvashskoy gosudarstvennoy selskokhozyaystvennoy akademii, 2018, No. 1 (4), pp. 93-96. (In Russian)

4. Putintsev S.V., Ageev A.G. Proverka effektivnosti primeneniya reber zhestkosti yubki porshnya malorazmernogo dizelya. Traktory $i$ selkhozmasiny, 2016, No. 11, pp. 35-39. (In Russian)

5. Sazgetdinov K.M., Berezovsky A.B., Maksimov A.V. Perspektivnye sistemy upravleniya gazoraspredeleniem avtomobilnykh dvigateley. Proceedings of XXIII Tupolevskie chteniya (shkola molodykh uchenykh), 2017, pp. 240-246. (In Russian)

6. Shtain G.W., Panfilov A.A. Ekspluatatsiya transportno-tekhnologicheskikh mashin $v$ zimnikh usloviyakh. Tyumen, TyumGNGU, 2016. 68 p. (In Russian)

7. Shtain G.W., Panfilov A.A. Mekhatron- 
тронная система энергетических установок современных автомобилей и транспортнотехнологических машин. Тюмень: ТИУ, $2018.90 \mathrm{c}$.

8. Эскин М.В. Разработка конструкции приспособления для контроля радиального и торцевого биений деталей типа "поршень" // Новые горизонты: материалы V Междунар. научно-практ. конф. 2018. С. 21-23.

9. Shtain G.V., Panfilov A.A. Experimental and computational research of the multicylinder engine crankshaft failure causes // International Journal of Mechanical and Production Engineering Research and Development. 2018. № 64. P. 537-548.

10. New HFO Common Rail System for Medium speed Diesel Engines. [Электронный pecypc]. - Режим доступа: http://docslide.us /documents/man-common-rail-cimac1.html.

11. Zhang X.M., Wang Y.Q., Fang J. Dynamic Simulation of Crank-Connecting RodPiston Mechanism of Internal Combustion Engine Based on Virtual Prototype Technology // Applied Mechanics and Materials. 2012. No. 143-144. P. 433-436.

DOI: $10.4028 /$ www.scientific.net/AMM.143144.433 . naya sistema energeticheskikh ustanovok sovremennykh avtomobiley $i$ transportnotekhnologicheskikh mashin. Tyumen, TIU, 2018. 90 p. (In Russian)

8. Eskin M.V. Razrabotka konstruktsii prisposobleniya dlya kontrolya radialnogo i tortsevogo bieniya detaley tipa "porshen". Proceedings of $V$ International conference "Novye gorizonty”, 2018, pp. 21-23. (In Russian)

9. Shtain G.V., Panfilov A.A. Experimental and computational research of the multicylinder engine crankshaft failure causes. International Journal of Mechanical and Production Engineering Research and Development, 2018, No.64, pp. 537-548.

10. New HFO Common Rail System for Medium speed Diesel Engines. Available at: http://docslide.us/documents/man-commonrail-cimac1.html

11. Zhang X.M., Wang Y.Q., Fang J. Dynamic Simulation of Crank-Connecting RodPiston Mechanism of Internal Combustion Engine Based on Virtual Prototype Technology. Applied Mechanics and Materials, 2012, No. 143-144, pp. 433-436.

DOI: $10.4028 / w w w . s c i e n t i f i c . n e t / A M M .143-$ 144.433. (In English) 